\title{
Hereditary Endometrial Cancer: Lynch Syndrome
}

\author{
Kouji Banno • Iori Kisu • Megumi Yanokura • \\ Kiyoko Umene • Yuya Nogami • Kosuke Tsuji • \\ Kenta Masuda • Arisa Ueki • Yusuke Kobayashi • \\ Wataru Yamagami • Nobuyuki Susumu • Daisuke Aoki
}

Published online: 9 November 2012

(C) Springer Science+Business Media New York 2012

\begin{abstract}
Lynch syndrome (hereditary nonpolyposis colorectal cancer [HNPCC]), Cowden syndrome (CS), and Peutz-Jeghers syndrome (PJS) are hereditary diseases with an increased risk for endometrial cancer. Lynch syndrome is the most frequent disease associated with hereditary endometrial cancer. Lynch syndrome is autosomal dominant disorder caused by germ-cell mutation of DNA mismatch repair genes. Patients with Lynch syndrome have a higher risk of endometrial cancer compared with the general population. Thus, these patients and their families may develop malignant tumors, including colon and endometrial cancers. The lifetime risk of endometrial cancer in females with Lynch syndrome is particularly high (28-60\%). Lynch syndrome is a typical hereditary tumor associated with endometrial cancer, and elucidation of the oncogenic mechanism is important to understand the characteristics of endometrial cancer, including sporadic endometrial cancer. The Amsterdam II Criteria are used for screening for Lynch syndrome, but some cases of hereditary endometrial cancer do not meet these criteria (masked Lynch syndrome); therefore, patients with a suspected hereditary predisposition, including juvenile-onset and double cancer, should undergo genetic tests in addition to taking of a family history.
\end{abstract}

Keywords Endometrial Cancer $\cdot$ Lynch syndrome $\cdot$ Revised Amsterdam Criteria · DNA mismatch repair gene · hMLH1 .

K. Banno $(\bowtie) \cdot \mathrm{I}$. Kisu $\cdot$ M. Yanokura $\cdot \mathrm{K}$. Umene $\cdot$ Y. Nogami K. Tsuji $\cdot$ K. Masuda $\cdot$ A. Ueki $\cdot$ Y. Kobayashi $\cdot$ W. Yamagami $\cdot$ N. Susumu $\cdot$ D. Aoki

Department of Obstetrics and Gynecology, School of Medicine, Keio University, Shinanomachi 35,

Shinjuku-ku, Tokyo 160-8582, Japan

e-mail: kbanno@z7.keio.jp
hMSH2 · hMSH6 · Lower uterine segment · Epimutation · Gynecological cancer

\section{Introduction}

Most cases of endometrial cancer result from multistep carcinogenesis after changes from a normal endometrium to atypical endometrial hyperplasia, which is a precancerous lesion in type 1 endometrial cancer. This process is thought to be due to accumulation of mutations in cancer-related genes, including oncogenes, tumor-suppressor genes, and DNA mismatch repair (MMR) genes. The cancer thus develops in a multistage process. In contrast, if aberrant cancer-related genes are already present in germ cells, the lifetime risk for endometrial cancer increases, and approximately $5 \%$ of cases of endometrial cancer are thought to be caused by a genetic predisposition.

Lynch syndrome (hereditary nonpolyposis colorectal cancer [HNPCC]), Cowden syndrome (CS), and PeutzJeghers syndrome (PJS) are hereditary diseases with an increased risk for endometrial cancer. Lynch syndrome is the most frequent disease associated with hereditary endometrial cancer. Lynch syndrome also is associated with aberrant expression of MMR genes, which is important for understanding the carcinogenic mechanism and biological characteristics of sporadic endometrial cancers. Furthermore, patients with Lynch syndrome have a high risk of developing other diseases, including colon cancer. Therefore, screening and preventive measures are important for these patients and their family. In this review, we focus on Lynch syndrome and describe the latest findings for this disease, including the carcinogenic mechanism and screening methods. 


\section{Lynch syndrome and endometrial cancer}

Lynch syndrome is a typical familial tumor with autosomal dominant inheritance. Female patients with Lynch syndrome complicate with endometrial cancer at a high incidence. In the revised 1999 Amsterdam II Criteria (ACII), endometrial cancer was included as a cancer similar to colon, small intestine, ureteral, and kidney cancers (Table 1) [1]. The prevalence of Lynch syndrome is $0.9-2.7 \%$ [2], and approximately $2.3 \%$ of cases of endometrial cancer occur due to Lynch syndrome [3]. The lifetime risk of endometrial cancer is $28-60 \%$ in females with aberrant genes associated with Lynch syndrome (Table 2) [4].

Lynch syndrome is caused by germ-cell mutation of DNA MMR genes. Six MMR genes have been cloned: $h M L H 1$, hMSH2, hMSH3, hMSH6, PMS1, and PMS2. hMLH1, $h M S H 2$, and $h M S H 6$ mutations are particularly important in families of patients with Lynch syndrome (Table 3). Most mutations occur in $h M L H 1$ and $h M S H 2$ in patients with colon cancer [5], whereas $h M H S 6$ mutations are important role in tumorigenesis in patients with endometrial cancer $[6,7 \bullet]$. Aberrations in these MMR genes prevent correct repair of mismatched bases, resulting in DNA strands with different lengths (Figure 1). This phenomenon occurs in microsatellite regions of the human genome and is referred to as microsatellite instability (MSI). Most short-tandem repeats (STRs), such as microsatellites, are present in noncoding regions and do not produce proteins with mutations. However, some STRs can occur in regions coding genes for which mutation is involved in carcinogenesis, including BAX, which is related to apoptosis induction, and TGFRII, which is associated with inhibition of cell proliferation [7•].

\section{Clinicopathological characteristics of endometrial cancer in patients with Lynch syndrome}

Broaddus et al. [8] described the clinicopathological characteristics of 50 patients with Lynch syndrome-related endometrial cancer with an $h M S H 1$ or $h M S H 2$ mutation
Table 2 Risks of patients with hereditary diseases developing endometrial cancer throughout life

\begin{tabular}{ll}
\hline Disease & Risk throughout life \\
\hline Lynch syndrome & $42 \%$ (diseased until 70 years old) \\
& $61 \%$ (MSH2 mutation) \\
& $42 \%$ (MLH1 mutation) \\
Cowden syndrome & $5-10 \%$ \\
Peutz-Jeghers syndrome & $9 \%$ \\
\hline
\end{tabular}

(Group A), 42 patients with sporadic endometrial cancer aged $<50$ years with no aberrant mutation (Group B), and 26 patients with loss of hMLH protein expression and MSIpositive sporadic endometrial cancer due to $h M L H$ promoter hypermethylation (Group C). The mean ages in Groups A, $\mathrm{B}$, and $\mathrm{C}$ were $46.8,39.9$, and 61.1 years, with a significant difference between Groups A and C. There was a slightly more frequent occurrence of nonendometrioid adenocarcinoma (clear cell adenocarcinoma, serous adenocarcinoma, and carcinosarcoma) in Group A (14 \%). Regarding differentiation, G2 and G3 endometrial cancer cells were frequently detected in Group $\mathrm{C}$ and vascular invasion also was found in Group C. The International Federation of Gynecology and Obstetrics (FIGO) surgical stage III and IV advanced cancer was more common in Group B compared with Group A [8]. Thus, cases of MSI-positive sporadic endometrial cancer due to $h M L H 1$ promoter methylation had less differentiation and most were advanced cancer; in contrast, Lynch syndrome-related endometrial cancer due to $h M L H 1$ or $h M S H 2$ mutation was clinicopathologically similar to endometrial cancer with onset at an early age.

The cases of Lynch syndrome-related endometrial cancer in Broaddus et al. [8] included multiple histological types, including nonendometrioid adenocarcinoma, and had different characteristics from those of sporadic endometrial cancer. However, most patients with Lynch syndrome had $h M S H 2$ mutations and the clinicopathological characteristics may differ from those of patients with $h M L H 1$ mutations. In addition,
Table 1 Amsterdam Criteria in 1990 and 1999
Amsterdam Minimum Criteria (1990)

1. Al least 3 cases of colorectal cancer in relatives (verified pathologically)

2. One is a first degree relative to the other two

3. At least two successive generations should be affected

4. One case of colorectal cancer diagnosed before the age of 50 years old

5. FAP should be excluded

Revised Amsterdam Criteria II (1999)

1. At least 3 relatives with an HNPCC-associated cancer

(cancer of the colorectum, endometrium, small bowel, ureter or renal pelvis)

2-5: As for the minimum criteria 
Table 3 MMR genes related to Lynch syndrome

\begin{tabular}{lllll}
\hline Gene & Chromosomal position & cDNA size & Exon size & Gene size \\
\hline MSH2 & $2 \mathrm{p} 21$ & $2.8 \mathrm{~kb}$ & 16 & $73 \mathrm{~kb}$ \\
$M L H 1$ & $3 \mathrm{p} 21-\mathrm{p} 23$ & $2.3 \mathrm{~kb}$ & 19 & $58-100 \mathrm{~kb}$ \\
$M L H 3$ & $14 \mathrm{q} 24.3$ & $4.3 \mathrm{~kb}$ & 12 & $37 \mathrm{~kb}$ \\
$M S H 6$ & $2 \mathrm{p} 21$ & $4.2 \mathrm{~kb}$ & 10 & $20 \mathrm{~kb}$ \\
PMS1 & $2 \mathrm{q} 31$ & $2.8 \mathrm{~kb}$ & 12 & $93 \mathrm{~kb}$ \\
PMS2 & $7 \mathrm{q} 22$ & $2.6 \mathrm{~kb}$ & 15 & $16 \mathrm{~kb}$ \\
\hline
\end{tabular}

the prognosis of Lynch syndrome-related endometrial cancer does not differ from that of sporadic endometrial cancer [9], but the onset age of sporadic endometrial cancer has been found to be 60 years, whereas that of Lynch syndrome-related endometrial cancer is only 48 years [10].

The Committee for Gynecologic Cancer of the Japan Society of Obstetrics and Gynecology (JSOG) evaluated the patient background, clinicopathological factors, and the incidence and type of double cancer in 34 patients with Lynch syndrome-related endometrial cancer meeting ACII, through comparison with 873 of 2,457 Japanese patients with sporadic endometrial cancer as controls. The mean ages of the patients with Lynch syndrome-related and sporadic endometrial cancer were 49.9 and 56.9 years, respectively, with a significantly younger age and juvenile onset in Lynch syndrome-related cancer. Regarding tissue type, nonendometrioid adenocarcinoma was not found in patients with Lynch syndrome-related cancer, which differs from reports outside Japan. The differentiation rates in the Lynch syndrome-related and sporadic endometrial cancer patients were $73.5 \%$ and $59.3 \%$ in G1, $8.8 \%$ and $24.2 \%$ in G2, and $17.7 \%$ and $15.1 \%$ in G3, respectively, indicating greater occurrence of the well-differentiated type in the Lynch syndrome-related group. The FIGO surgical stage was Stage
I in $85.3 \%$ of the patients with Lynch syndrome-related endometrial cancer and $66.5 \%$ in those with sporadic endometrial cancer group, showing a greater occurrence of Stage I cases in Lynch syndrome-related endometrial cancer. The incidences of double cancer in the Lynch syndromerelated and sporadic endometrial cancer patients were $38.2 \%$ and $5.8 \%$, respectively, with a significantly higher incidence in Lynch syndrome-related cancer. Regarding the type of double cancer, colon cancer was present in $69.2 \%$ of the cases of Lynch syndrome-related endometrial cancer, whereas ovarian cancer, colon cancer, and breast cancer were found in $35.3 \%, 25.5 \%$, and $23.5 \%$ of the cases of sporadic endometrial cancer, respectively [11]. These reports suggest that the characteristics of Lynch syndrome in Asia may differ from those in Europe and the United States. International accumulation of cases and analyses are needed to resolve this issue [7•].

\section{Hereditary endometrial cancer not meeting the Amsterdam II Criteria (ACII)}

The revised 1999 Amsterdam II Criteria (ACII) is used for clinical screening of Lynch syndrome. However, the results
Fig. 1 Clinical criteria of Lynch syndrome

\section{Single-based mismatch}

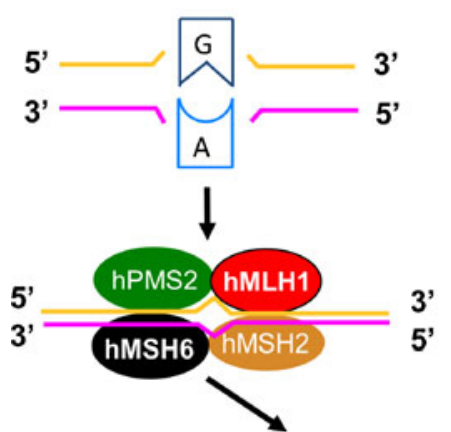

Repeat mismatch

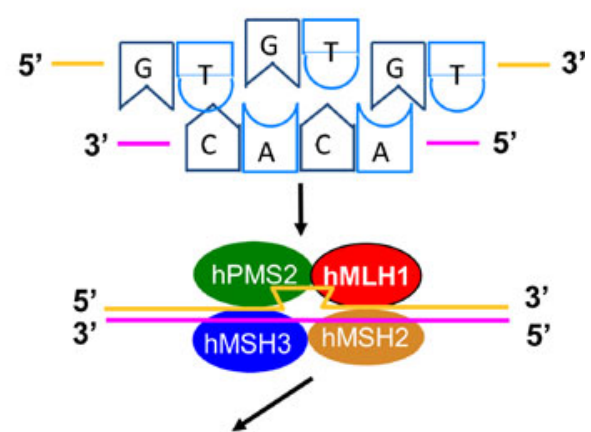

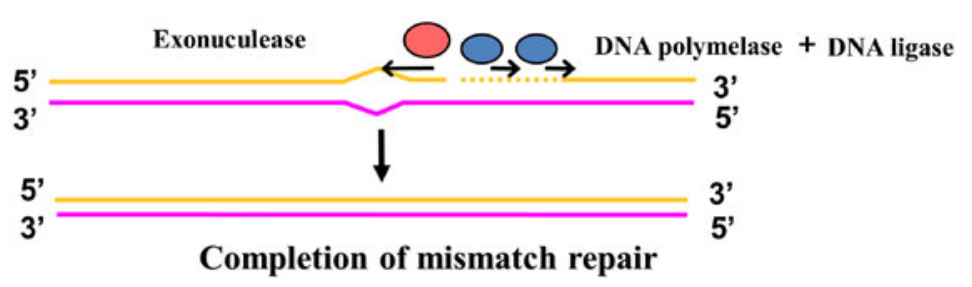


of germline MMR gene mutations show that some patients with a mutation do not meet ACII. These cases are referred to as cryptic Lynch syndrome. Thus, the incidence of Lynch syndrome-related endometrial cancer may actually be higher. Hampel et al. conducted a genetic analysis of MMR genes in 543 patients with endometrial cancer and detected a mutation in ten $(1.8 \%)$ of these cases (1 with $h M L H 1,2$ with $h M S H 2$, and 6 with hMSH6), of which seven did not meet ACII [12]. ACII does not include Lynch syndrome-related cancers, such as gastric, breast, and ovarian cancers, and therefore these diagnostic criteria alone cannot detect all cases of familial endometrial cancer.

Hirai et al. performed mutation analysis of germline genes in patients with frequent familial onset of Lynch syndrome-related tumors (Group A) and double cancer (Group B). The multicenter collaborative study included patients with at least two first-degree relative probands (including themselves) with Lynch syndrome-related tumors (colon, endometrial, small intestinal, ureteral, renal pelvic, gastric, breast, and ovarian cancers) and in whom the age at onset of at least one tumor was $\leq 50$ years in Group A, and patients with synchronous or metachronous double Lynch syndrome-related tumors who met the criteria in the revised Bethesda Guidelines for Lynch syndrome (2004) regardless of the onset age in Group B. Peripheral blood leukocyte-derived DNA was obtained from 120 patients (Group A: 57; Group B: 48; and Groups $\mathrm{A}+\mathrm{B}$ : 15) and germline mutations in $3 \mathrm{MMR}$ genes (hMLH1, hMSH2 and hMSHO) were analyzed by direct sequence analysis. Gene mutations were found in $18(15 \%)$ of the 120 patients: $9(15.8 \%)$ in Group A; 4 $(8.3 \%)$ in Group B; and 5 (33.3\%) in Groups A+B. Of 18 patients with genetic mutations, $5(27.8 \%)$ had a mutation in $h M L H 1,4(22.2 \%)$ in $h M S H 2$, and 9 $(50 \%)$ in $h M S H 6$. Of patients with mutations in hMSH6, eight had mutations mainly in exons 4-6 and most were frameshift mutations. Two of four patients who met ACII criteria (1 in Group A and 3 in Groups A+B) had $h M L H 1$ and $h M S H 2$ mutations, respectively. Among patients with double cancer who had genetic mutations, of six patients with double cancer, including colon cancer, five and one had mutations in $h M L H 1$ and $h M S H 2$, respectively. In contrast, all three patients with double cancer, including breast or ovarian cancer, had mutations in hMSH6. All of the double cancers developed simultaneously with or before development of endometrial cancer [13, 14]. These results suggest that there may be many cases of Lynch syndrome-related endometrial cancer and that genetic analyses for MMR genes are useful in patients with endometrial or double cancer showing a familial cluster of cancer. Furthermore, mutation of the hMSH6 gene appears to be particularly strongly associated with carcinogenesis of endometrial cancer.

\section{Screening and surveillance for Lynch syndrome-related endometrial cancer}

In general, hereditary tumors tend to be characterized by juvenile onset, multiple organ double cancer, and bilateral occurrence. The guidelines of the Gynecologic Oncologists (SGO) are useful for specific diagnosis of Lynch syndrome. These guidelines suggest that patients with endometrial and colon cancers who meet ACII, those who simultaneously or metachronously developed endometrial and colon cancers at age $<50$ years, those who simultaneously or metachronously develop ovarian and colon cancers at age $<50$ years, and those with a first or second-degree relative with aberrant MMR genes have a $>20-25 \%$ probability of hereditary predisposition for endometrial and colon cancers. Therefore, hereditary risks should be evaluated by genetic analysis in these patients. It has been shown that in patients diagnosed with endometrial and colon cancers at age $<50$ years, those with endometrial and ovarian cancers who simultaneously or metachronously complicate with a Lynch syndrome-related tumor, those with endometrial and colon cancers with at least two first- or second-degree relatives who developed a Lynch syndromerelated tumor, and those with a first- or second-degree relative who met the above criteria have a $>5-10 \%$ probability of hereditary predisposition for endometrial and colon cancers; therefore, evaluation of hereditary risks by genetic analysis is useful in these patients [15].

MSI analysis and immunohistochemical staining are useful for secondary screening to identify subjects who should undergo a mutation test for Lynch syndrome. The National Cancer Institute (NCI) recommends five satellite markers (BAT25, BAT26, D2S123, D5S346, and D173250) for MSI analysis [16]. A case with an allelic shift in at least two markers is defined as MSI-high and that with only one marker as MSI-low. However, most MSI reflects inactivity due to epigenetic changes in MMR genes and particularly MLH1 promoter methylation, which occurs in sporadic endometrial cancer at a rate of approximately $20 \%$ [17]. Therefore, MSI for screening for Lynch syndrome may not always be useful in patients with endometrial cancer. Immunohistochemical staining of MMR proteins may be less expensive and more effective for identifying aberrant MMR genes [18].

Patients with Lynch syndrome and persons from a family with Lynch syndrome have a high risk of other cancers. Therefore, appropriate screening and preventive measures for cancer are needed. Thus, Lindor et al. proposed that females with Lynch syndrome should undergo colonoscopy (every 1-2 years from age 20-25 years), transvaginal ultrasonography (once a year from age 30-35 years), urine cytology (every 1-2 years from age $25-35$ years), and history taking and physical examination, including genetic counseling and a system review (every year from age 21 years), with hysterectomy and adnexectomy considered 
if a patient does not want a child [19]. However, the effectiveness of these surveillance methods is unclear [20]. Preventive hysterectomy and ovariectomy have been discussed for risk reduction in colon cancer surgery for patients with Lynch syndrome who are older than reproductive age, but a consensus has not been reached.

\section{Lynch syndrome and endometrial cancer in the lower uterine segment}

Pathologically, the endometrium includes two regions: the uterine corpus (UC) and the lower uterine segment (LUS) [21]. Endometrial cancer usually develops in the mucosa of the UC and the uterine fundus but occasionally in the uterine isthmus in the LUS. If tumors are observed macroscopically to develop in the LUS and expand from the lower uterine corpus to the upper cervix, the disease is defined as carcinoma of the LUS. Cancer spreading from the uterine body to the internal cervix is excluded from this definition because the initial site is uncertain [22]. The LUS is located between the uterine body and cervix and has both endometrial and cervical characteristics in the grandular epithelium and stroma. The mucosal layer of the LUS is thinner compared with the uterine body, making the mucosal layer unlikely to respond to hormone stimulation [21]. Only a few cases have been described due to the rarity of endometrial cancer in the LUS; however, these cases have been shown to have clinicopathological characteristics that differ from those in normal endometrial cancer.

Westin et al. showed that 5 (14.2\%) of 35 patients with carcionma of the LUS met ACII criteria and had Lynch syndrome. All five patients had $h M S H 2$ mutation and also MSI, with decreased expression of hMSH2 protein shown by immunostaining. Among patients who did not meet ACII criteria, decreases in hMSH2 and hMSH6 protein levels were found in immunohistochemical staining and ten patients $(29 \%)$ were considered to have Lynch syndrome, including four who were strongly suspected to have a hMSH2 mutation due to high MSI and one who had a decreased expression of hMLH1 protein, but no aberrant methylation [23]. These results show an incidence of Lynch syndrome in carcinoma of the LUS of $14.2 \%$ based on the five patients with a definitive diagnosis of Lynch syndrome and an extremely high incidence based on the 1-2\% incidence of Lynch syndrome in patients with general endometrial cancer. Using immunological staining, Garg et al. showed that 5 of 32 patients with decreased expression of MMR proteins (hMLH1, hMSH2, hMSH6, and PMS2) had carcinoma of the LUS, whereas only 1 of 39 patients without abnormal protein expression had this disease, indicating a relationship between Lynch syndrome and carcinoma of the LUS [24]. Masuda et al. examined nine patients with carcinoma of the LUS and found that the onset age was significantly younger than that in cases of non-LUS carcinoma, although there were no significant differences in tissue type, FIGO surgical staging and MSI. One of the nine patients $(11 \%)$ with carcinoma of the LUS met ACII criteria and had Lynch syndrome. These patients had mutations of $h M L H 1$, but not of $h M L H 2$, and also had MSI and decreased hMLH1 protein in immunostaining. These results show a strong relationship of Lynch syndrome with carcinoma of the LUS [25•].

\section{Epimutation and Lynch syndrome}

Epimutation is defined as abnormal transcriptional repression of active genes caused by epigenetic abnormalities in germ cells. Epimutation is thought to be a direct cause of oncogenesis. Methylation has been shown recently in DNA from normal tissues and peripheral blood in cases of sporadic colorectal cancer, and many studies have shown epimutation in cancers, including epimutation of the DNA MMR genes $h M L H 1$ and $h M S H 2$. These results have led to a focus on epimutation as a novel oncogenic mechanism [26 ].

Epimutation affects one or both alleles of a gene and effectively reduces the level of the gene product by preventing transcription of the affected allele. Germline epimutation is an event in which epigenetic changes, such as aberrant methylation, occur at the stage of germ cells and are maintained in fertilization and embryonic development, resulting in persistence in adult somatic cells. Epigenetic characteristics can be transmitted over generations, but epimutation is not always inherited and may have a hereditary form that does not follow Mendelian inheritance [26•, 27-30]. Epimutation also may completely disappear in spermatogenesis [31], but epimutation inherited from the mother alone is unlikely to disappear during oogenesis [30, 31].

The relationship between epimutation and cancer has mainly been analyzed in colon cancer. Gazzoli et al. first showed that $h M L H 1$ may be methylated in peripheral blood, as well as in tumor tissue, in patients with colorectal cancer [32], based on a study in 14 patients with suspected Lynch syndrome with MSI. None of these patients had methylation of MMR genes, but hypermethylated $h M L H 1$ (approximately $50 \%$ ) was found in normal blood DNA in one 25-yearold female patient. Allele methylation in tissues derived from the embryologically discrete germ layer in this patient suggested that the methylation pattern may be constitutional or germline methylation. Because no mutation was found in specimens from her parents, hereditary evidence for epimutation was not obtained; however, it is notable that methylation occurred in such a young patient.

Patients with multiple cancers also have been shown to have $h M L H 1$ epimutation [33]. $h M L H 1$ methylation was 
found in a phenotype derived from a triploblastic organism in two patients with multiple cancer. Tissues from the parents of these patients were not examined, but no methylation was found in tissues in four of their five children. Much controversy exists regarding constitutional epimutation, including whether this is transmitted from a mother or father or occurs de novo in early embryonic development. Miyakura et al. found methylation in the $h M L H 1$ promoter region in peripheral blood lymphocytes in 4 of 30 patients with juvenile-onset sporadic colon cancer or multiple cancer, and one of the four patients had multiple cancer, including endometrial cancer [34]. Methylation was found only in one allele. Although it is unknown whether methylation is inherited, MSI was found in all patients and methylation also was detected in normal tissues of the large intestine, digestive mucosa, endometrium, and bone marrow in three patients. It is of interest that this finding and loss of heterozygosity (LOH) in both alleles of $h M L H 1$ in colon cancer are consistent with the mechanism of carcinogenesis by germline epimutation proposed by Suter et al., based on Knudson's "two hit" hypothesis (Figure 2).

Thus, a new type of Lynch syndrome has been found in patients with clinically suspected Lynch syndrome. This new type has no pathologic mutations in MMR genes but is characterized by epimutation in the promoter region of $h M L H 1$ [35]. This finding suggests that germline epimutation in MMR genes is a cause of Lynch syndrome. Additionally, in families with $h M S H 2$ methylation, germline mutation of epithelial cell adhesion molecule (EPCAM) is a cause of epimutation [36]. EPCAM is highly expressed in epithelial tissues and cancer, and a 3 ' end deletion in EPCAM causes read through with $h M S H 2$, which causes hypermethylation of $\mathrm{CpG}$ islands in the $h M S H 2$ promoter [37]. Furthermore, methylation of $h M S H 2$ in Lynch syndrome kindreds has been shown to be

Fig. 2 Based on Knudson's

"two hit" hypothesis transmitted genetically [35] and to follow Mendelian inheritance, in contrast to epimutation of $h M L H 1$. To date, only two families have been found to have hereditary epimutation of MMR genes; however, it is possible that epimutation of MMR genes is a factor in onset of hereditary endometrial cancer, similarly to colon cancer, and further studies are required to investigate this possibility.

\section{Cowden syndrome and endometrial cancer}

Cowden syndrome (CS) is a rare disease with autosomal dominant inheritance that is characterized by formation of multiple hamartomas in various tissues. The prevalence of CS is estimated to be 1 per $200,000-250,000$ population [38]. Patients with CS have increased morbidity risks for various malignant tumors, particularly breast, thyroid, and endometrial cancers. The PTEN (phosphatase and tensin homologue deleted on chromosome 10) gene is associated with onset of CS [39] and approximately $80 \%$ of patients with CS have a PTEN mutation [40]. The lifetime risk of endometrial cancer is $5 \%$ to $10 \%$ in patients with CS compared with $2 \%$ to $4 \%$ in the general population [41].

$P T E N$ codes for a protein with tyrosine kinase activity and acts as a tumor suppressor gene. PTEN also is a phosphatidylinositol phosphatase that regulates the reverse reaction of $\mathrm{PI} 3 \mathrm{~K}$ and inhibits activation of Akt via PI3K. Akt is a serine/threonine kinase that activates or inactivates downstream factors, with resulting transmission of signals related to cell growth, survival, differentiation, and glucose metabolism [42]. Akt is related to cell survival through inactivation of apoptosis executioners and transcription factors related to apoptosis-inducing factors. Therefore, if PTEN regulation of Akt activation is lost, the PI3K-Akt pathway is activated, and this leads to malignant

First hit Second hit

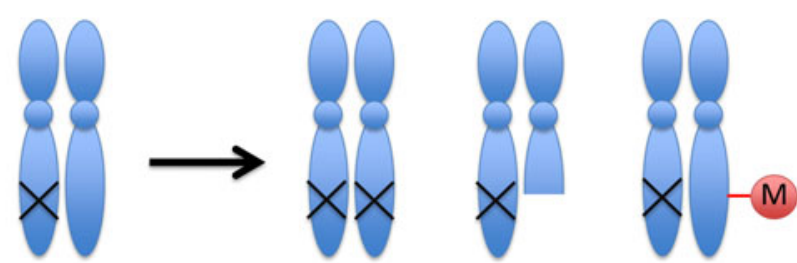

(B)

(A)

Mutation
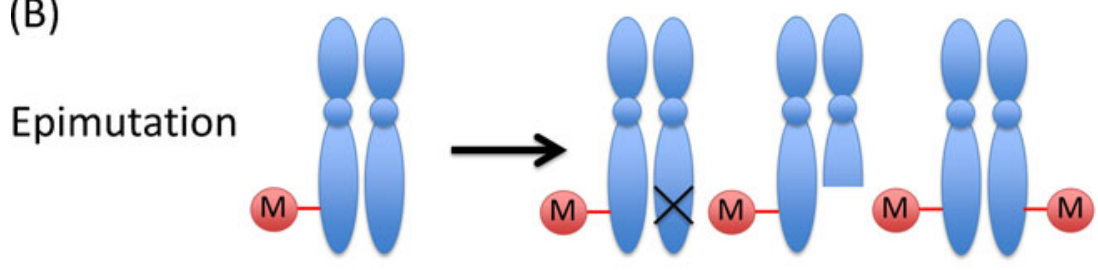

M- : Methylation 
transformation of cells. Regarding surveillance for endometrial cancer in patients with CS, cytology for women in their late $30 \mathrm{~s}$ and transvaginal ultrasonography for postmenopausal women have been proposed; however, the effectiveness of these surveillance methods remains unclear.

\section{Peutz-Jeghers syndrome and endometrial cancer}

Peutz-Jeghers syndrome (PJS) is characterized by multiple hamartomatous polyps in the gastrointestinal tract and mucocutaneous pigmentation. Patients with PJS have a higher risk of developing a malignant tumor in the gastrointestinal tract and other organs compared with the general population. LKB1/ STK11 has been identified as a disease-related gene with autosomal dominant inheritance. $L K B 1$ mutation is found in $80-94 \%$ of patients with PJS [43, 44]. The incidence of PJS is estimated to be 1 per 50,000-250,000 population [45]. Patients with PJS are at risk for gynecological cancers and have a $9 \%$ lifetime risk of developing endometrial cancer [46]. LKB1/ STK1 codes for a serine/threonine kinase that directly phosphorylates AMPK and has functions including regulation of glucose, lipid metabolism, cell proliferation, and cell polarity. PJS has less risk for endometrial cancer compared to Lynch syndrome and no specific surveillance is required.

\section{Conclusions}

Lynch syndrome, CS, and PJS are hereditary diseases associated with endometrial cancer, and Lynch syndrome is particularly important with regard to the prevalence and risk for developing endometrial cancer. In most patients with Lynch syndrome, endometrial cancer is likely to be a sentinel cancer; therefore, it is important to suspect Lynch syndrome if juvenile endometrial cancer is diagnosed. ACII criteria are used for surveillance of Lynch syndrome, but genetic analysis is important in high-risk patients with familial accumulation, even if they do not meet diagnostic criteria. For patients with a hereditary predisposition, screening and preventive measures are recommended, but the efficacy of these approaches is currently unknown. Surveillance for endometrial cancer also is recommended in patients with CS and PJS, but the risk of developing endometrial cancer is lower than that in Lynch syndrome and screening may be less effective. An investigation of the benefits of current screening and preventive measures is required, with establishment of new diagnostic criteria and surveillance methods for hereditary endometrial cancer.

Disclosure No potential conflicts of interest relevant to this article were reported.

\section{References}

Papers of particular interest, published recently, have been highlighted as:

- Of importance

1. Vasen HF, Watson P, Mecklin JP, Lynch HT. New clinical criteria for hereditary nonpolyposis colorectal cancer (HNPCC, Lynch syndrome) proposed by the International Collaborative group on HNPCC. Gastroenterology. 1999;116:1453-6.

2. Boland CR, Shike M. Report from the Jerusalem workshop on Lynch syndrome-hereditary nonpolyposis colorectal cancer. Gastroenterology. 2010;138:2191-7.

3. Hampel H, Panescu J, Lockman J, et al. Comment on: Screening for Lynch Syndrome (Hereditary Nonpolyposis Colorectal Cancer) among Endometrial Cancer Patients. Cancer Res. 2007;67:9603.

4. Meyer LA, Broaddus RR, Lu KH. Endometrial cancer and Lynch syndrome: clinical and pathologic considerations. Cancer Control. 2009;16:14-22.

5. Peltomäki P, Vasen H. Mutations associated with HNPCC predisposition - Update of ICG-HNPCC/INSiGHT mutation database. Dis Markers. 2004;20:269-76.

6. Schweizer P, Moisio AL, Kuismanen SA, et al. Lack of MSH2 and MSH6 characterizes endometrial but not colon carcinomas in hereditary nonpolyposis colorectal cancer. Cancer Res. 2001;61:2813-5.

7. • Banno K, Kisu I, Yanokura M, Masuda K, et al. Epigenetics and genetics in endometrial cancer: new carcinogenic mechanisms and relationship with clinical practice. Epigenomics. 2012;4:147-62. This is a recent overview of hereditary endometrial cancer.

8. Broaddus RR, Lynch HT, Chen LM, et al. Pathologic features of endometrial carcinoma associated with HNPCC: a comparison with sporadic endometrial carcinoma. Cancer. 2006;106:87-94.

9. Boks DE, Trujillo AP, Voogd AC, et al. Survival analysis of endometrial carcinoma associated with hereditary nonpolyposis colorectal cancer. Int J Cancer. 2002;102:198-200.

10. Vasen HF, Watson P, Mecklin JP, et al. The epidemiology of endometrial cancer in hereditary nonpolyposis colorectal cancer. Anticancer Res. 1994;14:1675-8.

11. Udagawa Y. Gynecologic Oncology Committee of the Japan Society of Obstetrics and Gynecology(JSOG): Hereditary endometrial cancer in Japan. Acta Obstetrica et Gynaecologica Japonica. 2009;61:1540-2.

12. Hampel H, Frankel W, Pnescu J, et al. Screening for Lynch syndrome (hereditary nonpolyposis colorectal cancer) among endometrial cancer patients. Cancer Res. 2006;66:7810-7.

13. Hirai Y, Banno K, Suzuki M, et al. Molecular epidemiological and mutational analysis of DNA mismatch repair (MMR) genes in endometrial cancer patients with HNPCC-associated familial predisposition to cancer. Cancer Sci. 2008;99:1715-9.

14. Banno K, Yanokura M, Kobayashi Y, et al. Endometrial cancer as a familial tumor: pathology and molecular carcinogenesis (review). Curr Genomics. 2009;10:127-32.

15. Lancaster JM, Powell CB, Kauff ND, et al. Society of Gynecologic Oncologists Education Committee statement on risk assessment for inherited gynecologic cancer predispositions. Gynecol Oncol. 2007;107:159-62.

16. Boland CR, Thibodeau SN, Hamilton SR, et al. A National Cancer Institute Workshop on Microsatellite Instability for cancer detection and familial predisposition: development of international criteria for the determination of microsatellite instability in colorectal cancer. Cancer Res. 1998;58:5248-57.

17. Esteller M, Levine R, Baylin SB, Ellenson LH, Herman JG. MLH1 promoter hypermethylation is associated with the microsatellite 
instability phenotype in sporadic endometrial carcinomas. Oncogene. 1998;17:2413-7.

18. Resnick KE, Hampel H, Fisher R, Cohn DE. Current and emerging trends in Lynch syndrome identification in women with endometrial cancer. Gynecol Oncol. 2009;114:128-34.

19. Lindor NM, Petersen GM, Hadley DW, et al. Recommendations for the care of individuals with an inherited predisposition to Lynch syndrome: a systematic review. JAMA. 2006;296:1507-17.

20. Vasen HF, Möslein G, Alonso A, et al. Guidelines for the clinical management of Lynch syndrome (hereditary non-polyposis cancer). J Med Genet. 2007;44:353-62.

21. Masuda K, Banno K, Yanokura M, et al. Carcinoma of the lower uterine segment (LUS): clinopathological characteristics and association with Lynch syndrome. Curr Genomics. 2011;12:25-9.

22. Jacques SM, Qureshi F, Ramirez NC, Malviya VK, Lawrence WD. Tumors of the uterine isthmus: Clinicopathologic features and immunohistochemical characterization of $\mathrm{p} 53$ expression and hormone receptors. Int J Gynecol Pathol. 1997;13:33-44.

23. Westin S, Lacour RA, Urbauer DL, et al. Carcinoma of the lower uterine segment: A newly described association with Lynch syndrome. J Clin Oncol. 2008;26:5965-71.

24. Garg K, Leitao Jr MM, Kauff ND, et al. Selection of endometrial carcinomas for DNA mismatch repair protein immunohistochemistry using patient age and tumor morphology enhances detection of mismatch repair abnormalities. Am J Surg Pathol. 2009;33:925-33.

25. - Masuda K, Banno K, Hirasawa A, et al.: Relationship of lower uterine segment cancer with Lynch syndrome: A novel case with a hMLH1 germline mutation. Oncol Rep 2012, In press. This is the second report on the relationship of LUS cancer and Lynch syndrome, and the first to describe an Asian patient with LUS cancer with Lynch syndrome induced by an hMLH1 germline mutation.

26. Banno K, Kisu I, Yanokura M, et al. Epimutation and cancer: a new carcinogenic mechanism of Lynch syndrome (review). Int $\mathbf{J}$ Oncol. 2012;41:793-7. This is a concise overview of the relationship between epimutation of MMR genes and Lynch syndrome.

27. Hitchins M, Williams R, Cheong K, et al. MLH1 germline epimutations as a factor in hereditary nonpolyposis colorectal cancer. Gasterenterology. 2005;129:1392-9.

28. Hitchins MP, Wong JJ, Suthers G, et al. Inheritance of a cancer-associated MLH1 germ-line epimutation. N Engl J Med. 2007;356:697-705.

29. Valle L, Carbonell P, Fernandez V, et al. MLH1 germline epimutations in selected patients with early-onset non-polyposis colorectal cancer. Clin Genet. 2007;71:232-7.

30. Morak M, Schackert HK, Rahner N, et al. Further evidence for hereditability of an epimutation in one of 12 cases with MLH1 promoter methylation in blood cells clinically displaying HNPCC. Eur J Hum Genet. 2008;16:804-11.
31. Hitchins MP, Ward RL. Erasure of MLH1 methylation in spermatozoa-implications for epigenetic inheritance. Nat Genet. 2007;39:1289.

32. Gazzoli I, Loda M, Garber J, Syngal S, Kolodner RD. A hereditary nonpolyposis colorectal carcinoma case associated with hypermethylation of the MLH1 gene in normal tissue and loss of heterozygosity of the unmethylated allele in the resulting microsatellite instability-high tumor. Cancer Res. 2002;62:3925-8.

33. Suter CM, Martin DI, Ward RL. Germline epimutation of MLH1 in individuals with multiple cancers. Nat Genet. 2004;36:497-501.

34. Miyakura Y, Sugano K, Akasu T, et al. Extensive but hemiallelic methylation of the hMLH1 promoter region in early-onset sporadic colon cancers with microsatellite instability. Clin Gastroenterol Hepatol. 2004;2:147-56.

35. Chan TL, Yuen ST, Kong CK, et al. Heritable germline epimutation of MSH2 in a family with hereditary nonpolyposis colorectal cancer. Nat Genet. 2006;38:1178-83.

36. Naruse H, Ikawa N, Yamaguchi K, et al. Determination of splicesite mutations in Lynch syndrome (hereditary non-polyposis colorectal cancer) patients using functional splicing assay. Fam Cancer. 2009;8:509-17.

37. Winter MJ, Nagtegaal ID, van Krieken JH, Litvinov SV. The epithelial cell adhesion molecule (Ep-CAM) as a morphoregulatory molecule is a tool in surgical pathology. Am J Pathol. 2003;163:2139-48.

38. Nelen MR, Kremer H, Konings IB, et al. Novel PTEN mutations in patients with Cowden disease: absence of clear genotypephenotype correlations. Eur J Hum Genet. 1999;7:267-73.

39. Nelen MR, Padberg GW, Peeters EA, et al. Localization of the gene for Cowden disease to chromosome 10q22-23. Nat Genet. 1996;13:114-6.

40. Liaw D, Marsh DJ, Li J, et al. Germline mutations of the PTEN gene in Cowden disease, an inherited breast and thyroid cancer syndrome. Nat Genet. 1997;16:64-7.

41. Salem OS, Steck WD. Cowden's disease (multiple hamartoma and neoplasia syndrome). A case report and review of the English literature. J Am Acad Dermatol. 1983;8:686-96.

42. Higuchi M, Onoshi K, Kikuchi C, Gotoh Y. Scaffolding function of PAK in the PDK1-Akt pathway. Nat Cell Biol. 2008;10:1356-64.

43. Ueki A, Kisu I, Banno K, et al. Gynecological tumors in patients with Peutz-Jeghers syndrome (PJS). OJGEN. 2011;1:65-9.

44. Beggs AD, Latchford AR, Vasen HF, et al. Peutz-Jeghers syndrome: a systematic review and recommendations for management. Gut. 2010;59:975-86.

45. Scully RE. Sex cord tumor with annular tubules a distinctive ovarian tumor of the Peutz-Jeghers syndrome. Cancer. 1970;25:1107-21.

46. Giardiello FM, Brensinger JD, Tersmette AC, et al. Very high risk of cancer in familial Peutz-Jeghers syndrome. Gastroenterology. 2000;119:1447-53. 